\title{
Real-Time Waste Controlling with ISB
}

\author{
M.Vasumathy, A.Punitha, R.P.Mahesh
}

\begin{abstract}
Nowadays, dustbins placed at the road are stuffed quickly due to the massive quantity of wastages in cities. Earlier waste controlling frameworks fundamentally dependent on the assortment of blended/arranged waste and moving it far to transfer zones has a critical negative effect on the earth and people. Wastages are generated from three varieties of sources like domestic, commercial and industrial. There is no quick replacement for the filled dustbin. This creates an unhealthful condition for all living things and spreads the bad smell around the streets. By doing so, there may be a probability to spread diseases. In order to avoid such situations, ISB (IOT based smart Bin) is to be implemented in cities of India. Smart Bin is to be placed at each street by tracking the level of garbage through the internet.
\end{abstract}

Keywords: Smart Bin, IoT, Waste Controlling, Garbage Collection, Arduino.

\section{INTRODUCTION}

Internet of Things (IoT) is a system of gadgets, for example, PC, workstation, tablet, vehicles, home appliances, and so on., installed with hardware, sensors, actuators, and network. This includes interfacing these sorts of gadgets through the web and offering their information. The dustbin is an essential requirement for each spot. It is seen that dustbin is filled inferable from the unpredictable evacuation of wastages. Since a large portion of the procedure is done through, the web should have a functioning rapid web association. This technology can be clarified as an association between human computers things. All the equipment's use in our everyday life can be controlled and observed utilizing the IoT. A larger part of the procedure is finished with the assistance of sensors in IoT. Sensors are employed over the place and these sensors convert basic physical information into advanced signals and transmit them to its control focus. By along these, the system can observe condition changes remotely from any piece of the world by means of web. The design of this framework would be founded on the set of tasks and procedures continuously situations.

Revised Manuscript Received on December 30, 2019.

* Correspondence Author

Dr.M.Vasumathy*, Associate Professor, Department of Computer Science, Madurai Kamraj University College, Madurai, India. Email: vasumathymohan@yahoo.co.in

Dr.A.Punitha, Assistant Professor, Department of Computer Science, N.M.S.S. Vellaichamy Nadar College, Nagamalai Pudukkottai, Madurai, India. Email: rp_ap@yahoo.com

Dr,R.P.Mahesh, Head and Assistant Professor, Department of Information Technology , N.M.S.S. Vellaichamy Nadar College, Nagamalai Pudukkottai, Madurai, India. Email: rp_ap@yahoo.com

(C) The Authors. Published by Blue Eyes Intelligence Engineering and Sciences Publication (BEIESP). This is an open access article under the CC BY-NC-ND license (http://creativecommons.org/licenses/by-nc-nd/4.0/)
Thinking about the need of current technology, the perception trash canister can costly yet considering the measure of dustbin required in India, costly trash container would not be an earlier examination that is the reasons have chosen to utilize spend the executive's framework utilizing IoT so as to decrease its cost and make it effective in applications. This ISB has been intended to pack the trash unit at standard interims of time by the assistance of the compaction unit to respond to the superfluous control of wastages.

\section{Implications \& Paper Organization:}

Hence, recommend that garbage collection models apply IoT Based Machine with Smart website for automated work easy for the user with ISB models. The rest of the paper is systematized as follows. Section II defines the review of the literature. Section III deliberates the proposed model. Finally, Section IV draws a conclusion.

\section{REVIEW OF LITERATURE}

As of late, individuals start utilizing technology for watching and gathering the damage in a productive methodology. The on-going investigation has been accomplished for productive IoT based keen containers for the clean condition [1] during which repository is given ultrasonic sensor and Arduino microcontroller with independent neighborhood shield for moving of information. The paper [2], addresses the instance of smart remote waste administration framework that uses the GSM revolution for smart bins. Chatterjee et al [3] utilized the Arduino microcontroller and with the assistance of CCTV cameras observed the earth. ShubhamThakker, R.Narayanamoorthi, during this paper [3], is utilizing the Near-Infrared reflectance (NIR) spectroscopy to distinguish the sort of plastic.

The distanced disperse gear from MSW (city strong waste) is place during a necessitous zone. By utilizing nonconformist materials which may be consolidated into a uniform material. The entire technique is rehashed every hour [4]. The paper [5] IOT based Garbage Managing System for brilliant Cities. Smart urban communities should be furnished with essential foundation and innovative headways to give the higher feel and com-post for living IOT based Garbage Managing System for Smart Cities" with the assistance of the Internet of Things (IoT) and cloud com frowning. Sharma, Narayan et al [6] built up a framework that defeats issues of trash bins being a flood and each one the refuse spills out prompting contamination. This likewise will build the number of diseases as a huge number of irritation and mosquitoes breed on it. Consequently, they planned a System dependent on AT89S52 for gathering the trash from a particular district - the locale whose open Garbage Bins are flooding with past concern. 
Vijayalaxmi [7] arranged a smart alert framework for trash leeway by giving an alarm sign to the city web server for prompt cleaning of a dustbin with legitimate confirmation dependent on the level of trash filling. This strategy is helped by the ultrasonic gadget which is interfaced with Arduino [10] UNO to analyze the degree of trash filled inside the dustbin and sends the aware of the city web server once the container is filled.

\section{PROPOSED SCHEME}

In the existing system, the corporation claims garbage. If this garbage overflows the bin and spread over the road, it will pollute the air and environment. Air pollution leads to various problems like asthma, Chronic Obstructive Pulmonary Disease (COPD), etc. Houseflies and mosquitoes mainly live in garbage. These are the major cause of various diseases like malaria, dengue, etc. Improper waste managing is found to be one of the major causes of human diseases causing premature death every year. ISB implementation will recover the lumping of garbage over a long period by preventing diseases largely and creating a clean environment in cities.

The framework under discussion assesses checking and an assortment of trash from waste receptacles set at various areas. Figure 1.1 shows the engineering of the proposed framework, where dustbin is outfitted with the ultrasonic sensor and that sensor is associated with the Arduino board having worked in Wi-Fi capacities to move sensor information to the trash-observing framework.

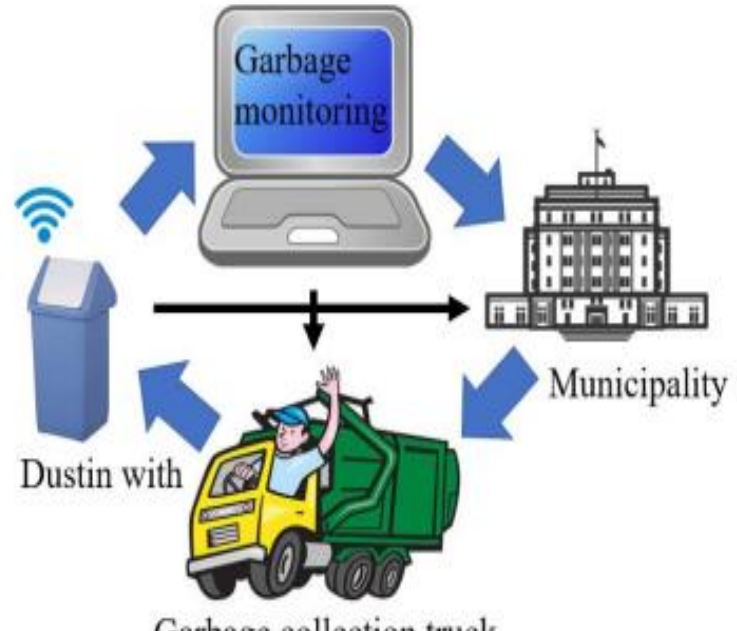

Garbage collection truck

Figure 1.1: General Flow of Garbage Collection

\subsection{ISB Model:}

The following figure shows the Layer of the ISB Model. This model is divided into four Layers such as Perception, Network, Middleware, and Application Layer.

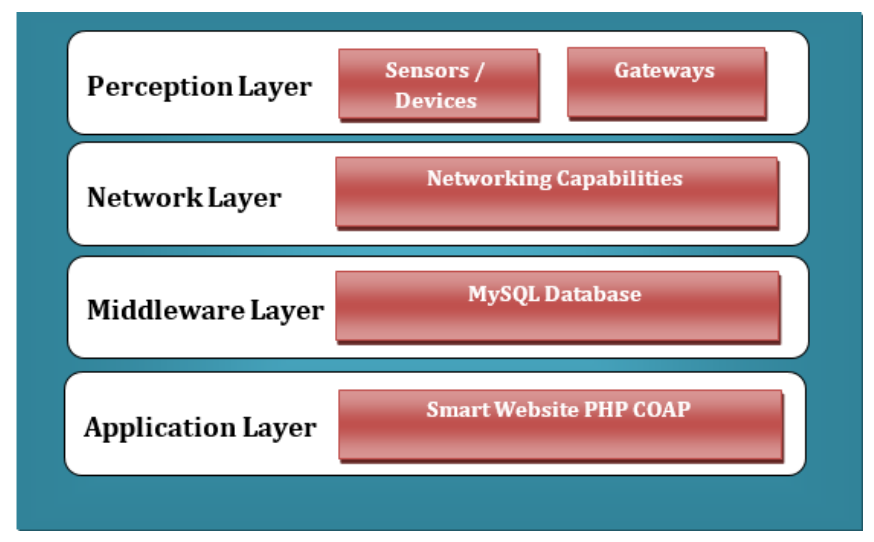

Figure 1.2: Layers of the ISB Model

\subsubsection{Perception Layer:}

The Smart Bin features include compaction unit, surveillance camera, energy utilization unit, level monitoring unit, Wi-Fi hotspot unit. These features will intimate the corporation about the level of garbage in Smart Bin.

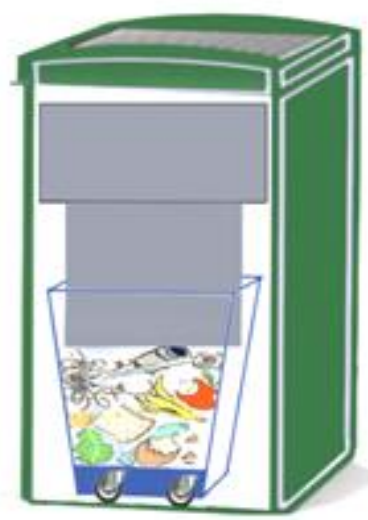

Figure 1.3: Compaction Unit

The lightweight garbage includes paper cups, plastic bags, which are generally occupied spaces in the dustbin. This does not consume the actual space of the dustbin. Therefore, if the dustbin is filled with lightweight particles, then the compaction unit starts compressing the waste up to a certain distance inside the dustbin with the help of a distance sensor. This can be done by several numbers of times. By doing this process, Smart Bin can store the garbage of four dustbins. This reduces the usage of normal dustbin at unnecessary places. Once the Smart Bin capacity is filled, it sends a message to the municipality to replace the bin immediately.

\section{A) Surveillance Camera Unit:}

Smart Bin contains a surveillance camera. This is utilized to watch the surroundings on the streets. Live video will be streaming by the surveillance camera. Mobile phones, tablets, etc can see the live video. It can separate objects by means of a camera and transfer the images to distributed storage. The Raspberry Pi Camera Module is a handcrafted adds on for Raspberry Pi [8]. It connects to Raspberry Pi by method for one of the two little attachments on the board upper surface. 
This interface utilizes the committed CSI interface, which was planned particularly for interfacing cameras. The Raspberry Pi Camera Module is a 5MP CMOS camera with a fixed lens focal point that is equipped for catching still pictures just as top quality video. Stills are caught at goals of $2592 \times 1944$, while video is supported at 1080p at $30 \mathrm{FPS}$, $720 \mathrm{p}$ at 60 FPS and $640 \times 480$ at 60 or 90 FPS [9]. The camera is reinforced in the most recent variant of Raspbian, Raspberry Pi's preferred operating system.

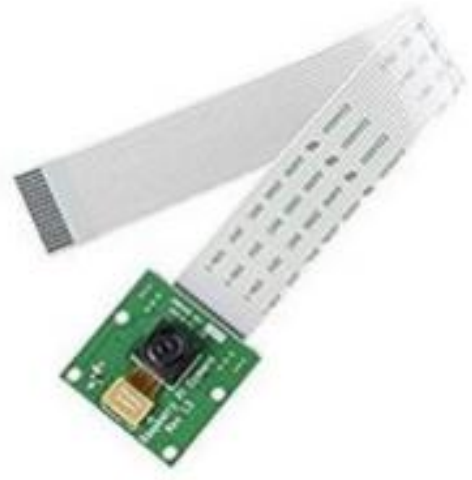

Figure 1.4: Raspberry Pi Camera

\section{B) Energy Utilization Unit:}

The power supply circuit comprises four rechargeable batteries, diodes, and solar panel battery charger. The positive terminal of the battery charger is associated with the positive terminal of the principal battery pair. The negative terminal of the battery charger is connected to the negative terminal of another pair of batteries.

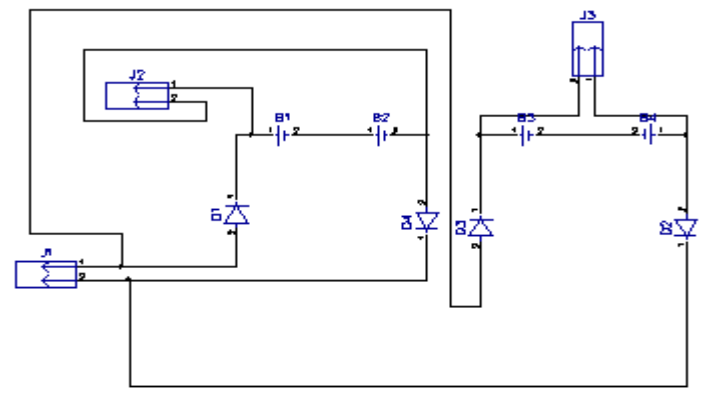

Figure 1.5: Power Supply Circuit

Solar panel power is delivered to the battery charger, whose output is given to the four batteries for recharging purposes. Diodes are utilized in this circuit. The key contribution of the diode is to halt the converse voltage from the batteries

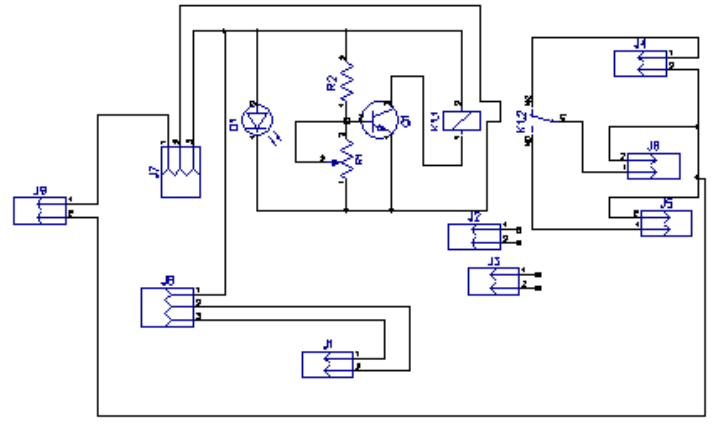

Figure 1.6: Control Circuit
The control circuit consists of the single-channel relay, transistor, Light Dependent Resistor (LDR), variable resistor and voltage regulators. This circuit controls the charging process of the battery with the help of Light Dependent Resistor (LDR) and single-channel relay. The control circuit contains two batteries, which are charged using a power control circuit.

In the relay, two sets of batteries are connected. The batteries will be charged based on the switching of the relay. This operation achieved by the usage of LDR. The transistor acts as a shift switch for the relay. In that transistor, the collector is related to the coil of the relay. The emitter terminal is grounded. The base terminal is associated with the voltage divider circuit.

The voltage divider circuit consists of a variable resistor and Light Dependent Resistor (LDR). Voltage regulators are utilized in this circuit. The main purpose of the voltage regulator is to regulate the unregulated input voltage to a regulated output voltage. When the LDR glows, the relay will be in Commonly Open condition. The first set of batteries will be charged. The second set will be operating. When the LDR does not glimmering, the converse operation will be accomplished by relay. The load can be satisfied with its required using this process.

\section{C) Level Monitoring Unit:}

The problem with the previous dustbin is that they can store up to a certain limit. When it is filled full of garbage, this is replaced by corporation workers once a day or week. There might be a chance to overflow garbage from the dustbin and spread across the road when the filled dustbin not yet replaced by an empty dustbin. As mentioned earlier, Smart Bin has the capacity of storing four garbage cans. The garbage can is also placed inside the Smart Bin to avoid overflowing garbage from the bin. Even though the smart bin is filled, this will send a message to the municipality.

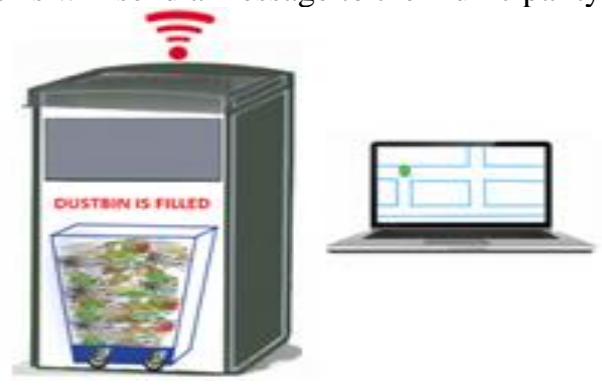

Figure 1.7: Level Monitoring

Ultrasonic transducers convert ultrasound waves to an electrical signal or the other way around and get may likewise be known as ultrasound handsets; a few ultrasound sensors other than being sensors are so handsets since the two of them will detect and transmit. These gadgets take a shot at a standard simply like that of transducers used in radar and sonar frameworks that assess traits of an objective by deciphering the echoes from radio or sound waves, severally. Dynamic ultrasonic sensors create high-recurrence sound waves and assess the reverberation that got back by the sensor, estimating the time interim between sending the sign and getting the sound to decide the distance to an item. The distance can be determined to utilize the equation: 


$$
\operatorname{Distance}(L)=\sum \frac{1}{2 * T * C}
$$

Where $\mathrm{L}$ denotes the distance of the object from the sensor, $\mathrm{T}$ represents the time between emission and reception, and $\mathrm{C}$ is the speed of the ultrasonic wave.

\section{D) Fire Detection Unit:}

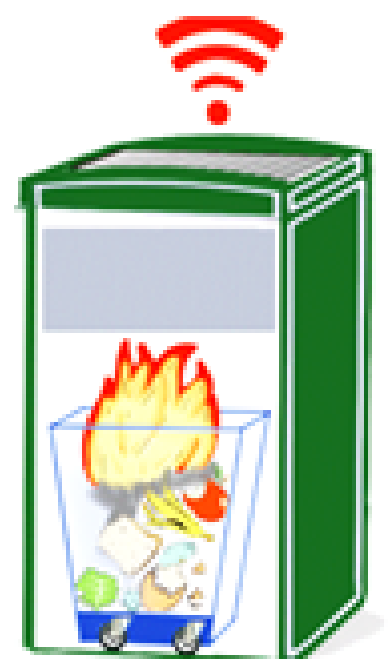

Figure 1.8: Fire Detection Unit

It is easy to capture fire on dustbins located on the streets. This generates air pollution and spoils the environment. Air pollution causes headaches, toxic gases and increases the stress level. Even smelling is a vital reason for various problems. When garbage burns inside the smart bin, this smoke or gas sensor indicates the municipality immediately. Then, the compaction unit will start to stop the fire. The sensor utilized in this framework is gas sensor QM-NG-1 to distinguish the nearness of smoke. It very well may be put inside the unit, yet it is best put at the highest point of the unit. The choice of the sensor was created because of its high affectability for noxious gas, long inductive time and long life expectancy.

\section{E) Wi-Fi Hotspot Unit:}

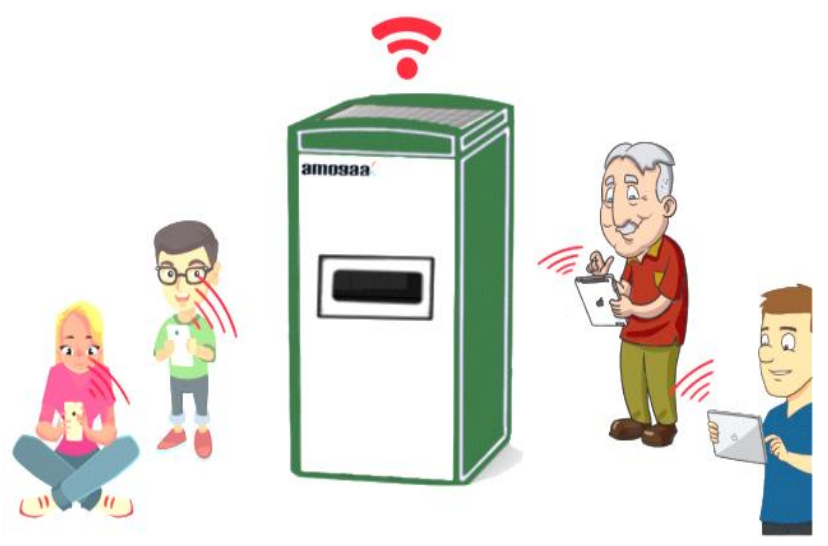

Figure 1.9: Wi-Fi Hotspot

This Smart bin has an inbuilt Wi-Fi router. The determination of $\mathrm{Wi}-\mathrm{Fi}$ is to send and obtain messages, photos, and videos to a certified person. People may also access this Wi-Fi free. They can watch video streaming via Wi-Fi.

\subsection{Network Layer}

The network layer is responsible for transmitting the deliberate data in the recognition layer to the upper layers, where the handling frameworks are arranged. What's more, utilizes ZigBee, Z-wire, GSM, UMTS, Wi-Fi, Infrared, 6LoWPAN. Notwithstanding the essential assignments, the system layer likewise accomplishes the distributed computing process and the information the boarding procedure.

\subsection{Middleware Layer}

The middleware layer is a layer of programming or even a lot of sub-layers that work to interrelate parts of the IoT that would not be possible to interconnect something else, that is, a translator. Notwithstanding giving simultaneousness so the application layer can cooperate with the layer of recognition and guarantee compelling correspondence, it has an essential impact on the advancement of innovations. In middleware, MYSQL Database is utilized to store the data from the sensor.

\subsection{Application Layer}

The application layer does not straightforwardly add to the improvement of an IoT structure, anyway it is in this layer and places the different services have planned that interface with clients, that is, any place the translation and accessibility of the data occur. Here form normal IoT Platform for each ISB Device should get to this. The COAP is an application layer convention created to help applications inside IoT frameworks. In view of the Representational State Transfer (REST) functionalities over HTTP, REST might be a vehicle convention utilized in systems with low power hubs, versatile applications, and informal communities, having the option to move information between customer and server in a more straightforward strategy, also to being a reserved association convention. In contrast to REST, COAP is associated with User Datagram Protocol (UDP) that makes it a lighter and progressively worthy convention for IoT applications, containing adjustments of HTTP functionalities for low power utilization while working on joins inside the nearness of noise and packet loss.

\begin{tabular}{|c|c|}
\hline User Interface & Website \\
\hline Website URL & http://ggitinfo.com/iot/ \\
\hline Language & PHP \\
\hline Protocol & HTTP \\
\hline API & REST API \\
\hline
\end{tabular}

\section{Table 1.1 User Interface Information}

In this layer, each individual device has its own key as the non-public key to access channel within the web site. The accompanying figure 1.10 shows some channel list on the site. This strategy permits sensors, instruments, and sites to send information to the cloud in any place it is kept in either a private or an open channel. Sensors, or things, sensitive information and largely act locally. The proposed system stores information in private channels as a matter of course, anyway open channels will be utilized to impart information to other people. When information is during this System channel, the client will investigation and imagines it, compute new information, or connect with various gadgets. 


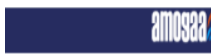

Public Channels
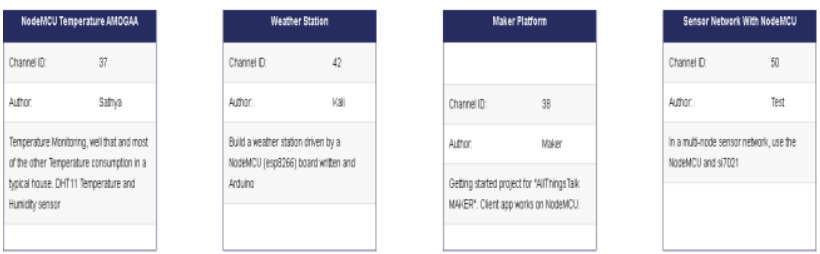

\section{Figure 1.10 Channel List}

This layer manages the entrance of things to the web and ensures they uncover their services through web API. This is regularly the center layer of the IoT as it guarantees things have an online available API, changing them into programmable things. The access layer inside the IoT is built around two center examples: as a matter of first importance, all things ought to uncover their administrations through a tranquil API. REST is a compositional style at the premise of the programmable web because of its usage in HTTP 1.1. As a result, if things supply restful APIs over HTTP, they get a URL and become consistently coordinated to the World Wide Web and its devices like programs, hyperlinked HTML pages, and JavaScript applications.

\section{RESULTS AND DISCUSSION}

This section of this work contains the results and its performance analysis for the smart bin in controlling the wastes. The analysis of the result based on the performance of the smart bin is described below with the illustrations.

The evaluation of CPU utilization rate for the ISB is shown in Figure 1.11. The figure depicts that the utilization of the CPU for the ISB is evaluated with respect to the requests transferred per min.

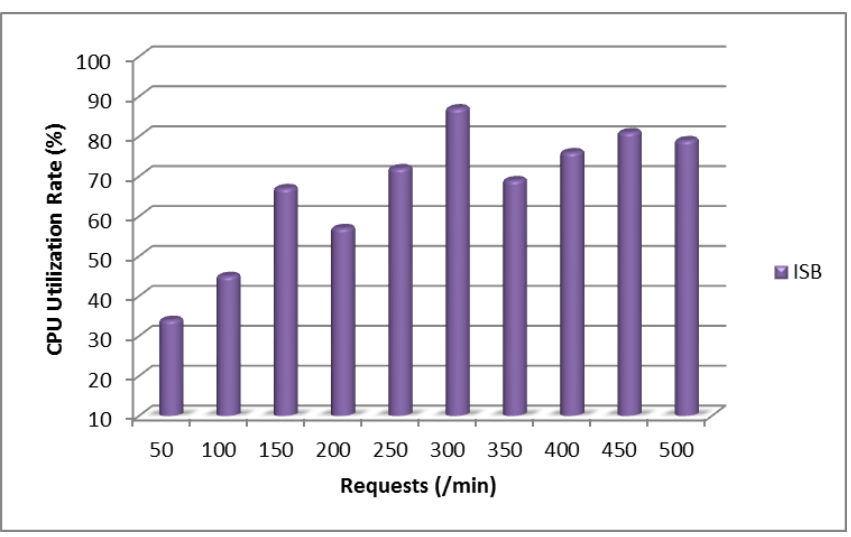

Figure 1.11 CPU Utilization Rate

The memory utilization for the ISB with respect to the respects / min is illustrated in Figure 1.12. The figure depicts that the utilization of memory while consuming the request transmission.

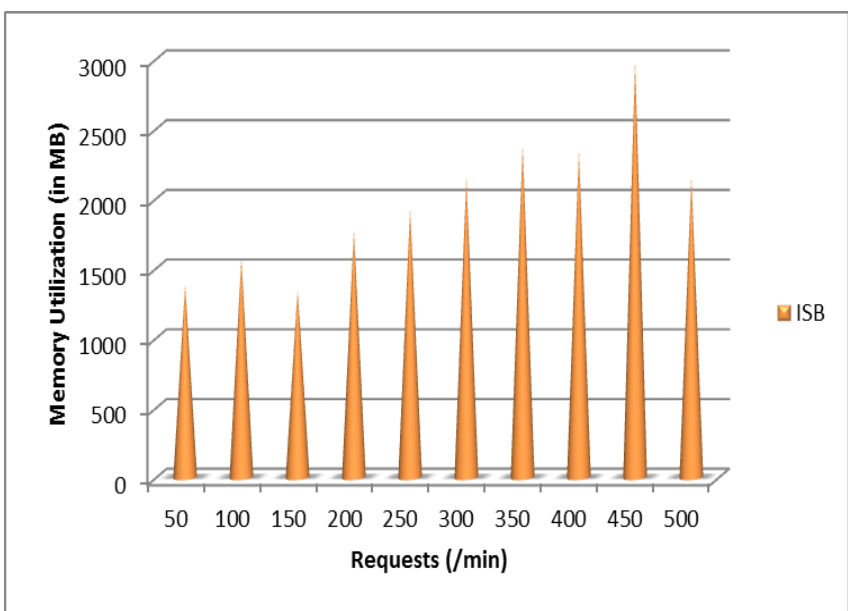

Figure 1.12 Memory Utilization

Figure 1.13 shows that the responding time taken by the ISB for the requests send to the server in $/ \mathrm{min}$. The figure shows that the ISB consumes less time for the response to the requests.

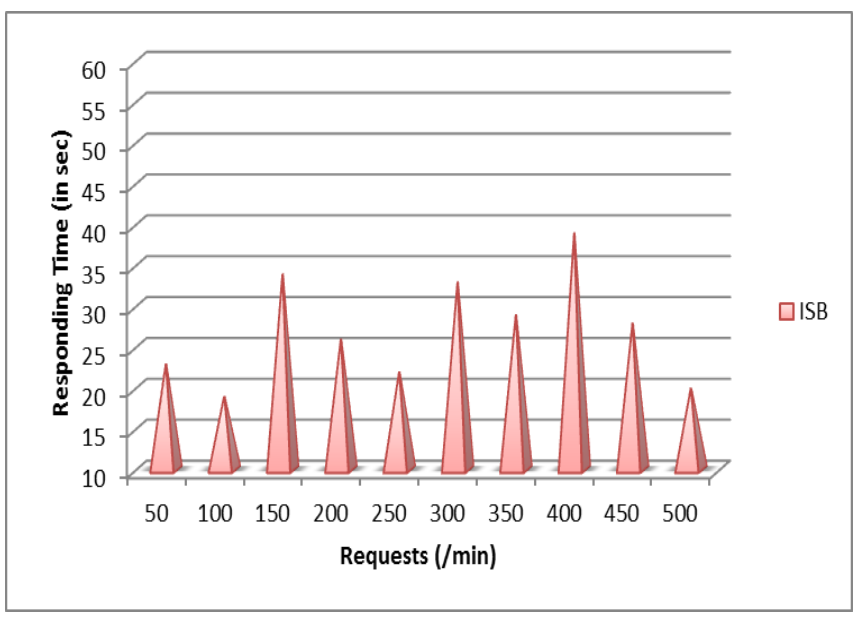

Figure 1.13 Responding Time for Requests

The following Table 1.2 shows tha result analysis based on the performance of the ISB.

\begin{tabular}{|c|c|c|c|}
\hline $\begin{array}{l}\text { Request } \\
\text { /min }\end{array}$ & $\begin{array}{l}\text { CPU } \\
\text { Utilization } \\
(\%)\end{array}$ & $\begin{array}{l}\text { Memory } \\
\text { Utilization } \\
\text { (in MB) }\end{array}$ & $\begin{array}{l}\text { Responding } \\
\text { Time(in sec) }\end{array}$ \\
\hline 50 & 34 & 1378 & 23 \\
\hline 100 & 45 & 1567 & 19 \\
\hline 150 & 67 & 1346 & 34 \\
\hline 200 & 57 & 1768 & 26 \\
\hline 250 & 72 & 1926 & 22 \\
\hline 300 & 87 & 2154 & 33 \\
\hline 350 & 69 & 2384 & 29 \\
\hline 400 & 76 & 2344 & 39 \\
\hline 450 & 81 & 2985 & 28 \\
\hline 500 & 79 & 2156 & 20 \\
\hline
\end{tabular}

Table 1.2 Result Analysis

Published By: 


\section{Real-Time Waste Controlling with ISB}

\section{CONCLUSION}

Smart Bin is the beginning to focus on a spotless and sterile condition by building a smart city. Trash clearing has become an enormous problem to each side of the world to the created nations with a bigger amount of populace. The amount of trash generation radically will increment, anyway, the administration frameworks are poor in a large portion of the regions. The individuals who are contented to require one stage extra for expanding the tidiness in their regarded regions frequently utilize the framework as a benchmark. Ultrasonic detecting component is being utilized during this framework to envision the fill level of the dustbins, anyway in future different sorts of detecting components are frequently utilized with the ultrasonic sensor to get a lot of exact outcomes and different sorts of information to require this method to an alternate level.

\section{REFERENCES}

1. Murugaanandam. S, Ganapathy. V and Balaji. R, "Efficient IOT Based Smart Bin for Clean Environment", International Conference on Communication and Signal Processing, April 3-5, 2018, India

2. Thakker, S.akker, S. and Narayanamoorthi, R, "Smart and wireless waste management", Innovations in Information, Embedded and Communication Systems (ICIIECS), International Conference, March 2015

3. Chatterjee, S. \&Kar, A.K. (2015). Smart Cities in Developing Economies: A Literature Review and Policy Insights. IEEE International Conference on Advances in Computing, Communications, and Informatics. Kochi, India, pp. 2335 - 2340.

4. Andrei Borozdukhin, Olga Dolinina and VitalyPechenkin, "Approach to the Garbage Collection in the Smart Clean City Project" in, Yuri Gagarin State Technical University of Saratov, Saratov, Russia 2016.

5. QiangDuan, et al., "A survey on service-oriented network virtualization towards convergence of networking and IoT", Transactions on Network and Service Management, IEEE, Vol. 9, No. 4, 2012, pp.373-392.

6. Sharma, Narayan, NirmanSingha, and Tanmoy Dutta. "Smart bin implementation for smart cities." International Journal of Scientific \& Engineering Research 6.9 (2015): 787-791.

7. Dr. N Satish Kumar, B. Vijayalaxmi: IoT based smart garbage alert system

8. https://www.raspberrypi.org/documentation/usage/camera/python/RE ADME.md

9. https://www.makeuseof.com/tag/raspberry-pi-camera-module

10. R.Mahalakshmi Priya, Dr.M.Vasumathi, "Fleet Automation using IoT Logistics", International Journal of Engineering and Advanced Technology (IJEAT) ISSN: 2249 - 8958, Volume-8 Issue-6, August 2019.

\section{AUTHORS PROFILE}

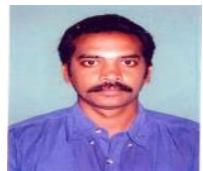

Dr.M.Vasumathy, M.Sc., MCA., M.Phil., Ph.D., Associate Professor, Department of Computer Science, MKU College, Madurai, India. She has 18 years of teaching experience, published six papers, and attended more than eight international conferences. Her research area is Software Engineering, Data Mining, and Image

Processing.

Dr.A.Punitha, B.Sc., MCA., M.Phil., Ph.D.

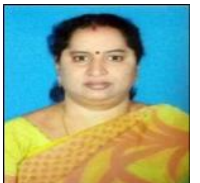

Assistant Professor, Department of Computer Science, N.M.S.S. Vellaichamy Nadar College, Nagamalai Pudukkottai, Madurai, India. She has 21 years of teaching experience, published three papers, and attended more than two international conferences. Her research area is Networking, Cloud Computing, and IoT.

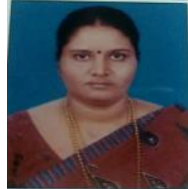

Dr.R.P.Mahesh, B.Sc., M.Sc.., M.Phil., Ph.D., Head and Assistant Professor, Department of Information Technology, N.M.S.S. Vellaichamy Nadar College, Nagamalai Pudukkottai, Madurai, India. He has 24 years of teaching experience; also, he published three papers and attended more than two international conferences. His research area is Networking, Data Mining, and IoT. 